\title{
Particle acceleration by relativistic expansion of magnetic arcades
}

\author{
Hiroyuki Takahashi ${ }^{1}$, Eiji Asano ${ }^{1}$ and Ryoji Matsumoto ${ }^{2}$ \\ ${ }^{1}$ Graduate School of Science and Technology, Chiba University, \\ 1-33 Yayoi-cho, Inage-ku, Chiba 263-8522, Japan \\ ${ }^{2}$ Department of Physics, Faculty of Science, Chiba University, \\ 1-33 Yayoi-cho, Inage-ku, Chiba 263-8522, Japan \\ email: takahasi,asano,matumoto@astro.s.chiba-u.ac.jp
}

\begin{abstract}
We carried out relativistic force free simulations and Particle In Cell (PIC) simulations of twist injection into the magnetic arcades emerging on the surface of a magnetar. As the magnetic energy is accumulated in the arcades, they expand self-similarly. In the arcades, a current sheet is formed and magnetic reconnection takes place. We also carried out 2-dimensional PIC simulations for the study of particle acceleration through magnetic reconnection. As a result, the energy spectrum of particles can be fitted by a power-law.
\end{abstract}

Keywords. acceleration of particles, magnetic field, plasmas, flare, neutron star

As a model of bursts of Soft Gamma-ray Repeaters (SGRs), Thompson \& Duncan (1995) proposed magnetic flares on the surface of a magnetar (see also Lyutikov 2006). To study the dynamical evolution of magnetic arcades, we carried out axisymmetric relativistic force free simulations. At the footpoints of a force-free magnetic arcade, shear motion is imposed with speed $10 \%$ of the light speed. After twist injection, fast waves propagate isotropically. Alfven waves propagating along the magnetic field lines transport magnetic energy from the the footpoints. As the magnetic energy is accumulated, they expand self-similarly. The Lorentz factor is about $3 \sim 4$ but locally exceeds 10 . Antiparallel magnetic field, and thus current sheets, are created inside the arcade. Magnetic reconnection can take place in this region. In front of the arcades, a fast shock is formed and particles can be accelerated by shock acceleration.

Next, we concentrated on the the reconnection region and carried out 2-dimensional relativistic PIC simulations to study particle acceleration. We assumed an electron-positron plasma. We also assumed that the plasma is collisionless and the plasma density is larger than the Goldreich-Julian density. By the dissipation of magnetic energy, particles are accelerated up to almost the potential energy difference given by shear motions. The energy spectrum of particles can be fitted by a power-law.

\section{Acknowledgements}

We would like to thank Dr. M. Hoshino and S. Shibata for useful discussions.

\section{References}

Lyutikov, M. 2006, MNRAS, 267, 1594

Thompson, C., \& Duncan, R. C. 1995, MNRAS, 275, 300 